Title: SIMULATED PERFORMANCE OF THE SUPERCONDUCTING SECTION OF THE APT LINAC UNDER VARIOUS FAULT AND ERROR CONDITIONS

DISTREUTION OF THS DOCLMENT IS UNLMTEO

\section{RECEIVED \\ AUG 141997 \\ 0.91}
Submitted To: 1997 Particle Accelerator Conference
Vancouver, BC, Canada
May 12-16, 1997
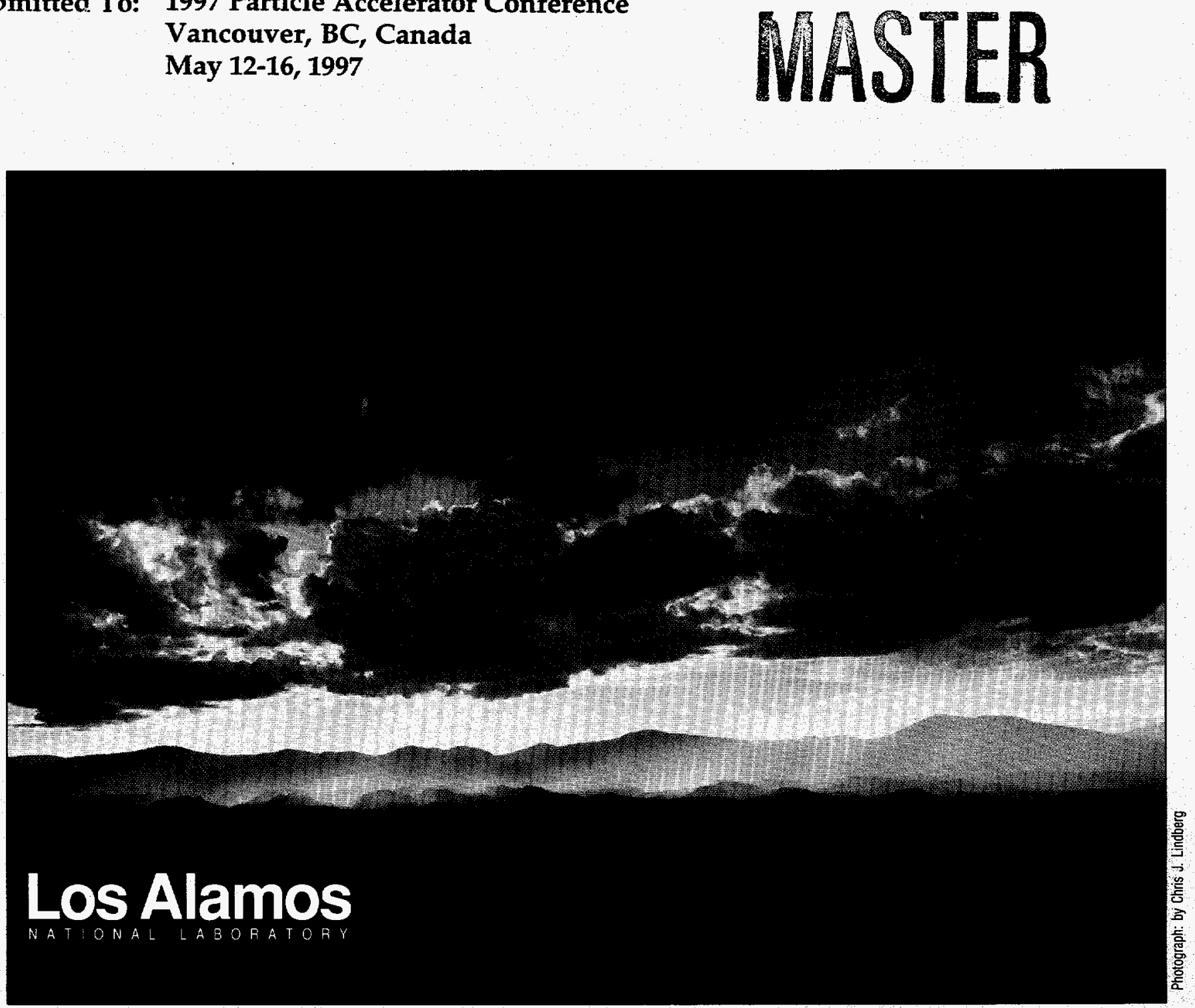

This is a preprint of a paper intended for publication in a journal or proceedings. Because changes may be made before publication, this preprint is made available with the understanding that it will not be cited or reproduced without the permission of the author. 


\section{DISCLAIMER}

This report was prepared as an account of work sponsored by an agency of the United States Government. Neither the United States Government nor any agency thereof, nor any of their employees, make any warranty, express or implied, or assumes any legal liability or responsibility for the accuracy, completeness, or usefulness of any information, apparatus, product, or process disclosed, or represents that its use would not infringe privately owned rights. Reference herein to any specific commercial product, process, or service by trade name, trademark, manufacturer, or otherwise does not necessarily constitute or imply its endorsement, recommendation, or favoring by the United States Government or any agency thereof. The views and opinions of authors expressed herein do not necessarily state or reflect those of the United States Government or any agency thereof. 


\section{DISCLAMVER}

Portions of this document may be illegible in electronic image products. Images are produced from the best available original document. 


\title{
SIMULATED PERFORMANCE OF THE SUPERCONDUCTING SECTION OF THE APT LINAC UNDER VARIOUS FAULT AND ERROR CONDITIONS*
}

\author{
E. R. Gray, S. Nath and T. P. Wangler \\ Los Alamos National Laboratory, Los Alamos, NM 87545
}

\begin{abstract}
The current design for the production of tritium uses both normal-conducting (NC) and super-conducting (SC) structures. To evaluate the performance of the superconducting part of the linac which constitutes more than $80 \%$ of the accelerator, studies have been made to include the effects of various error and fault conditions. Here, we present the simulation results of studies such as effects of rf phase and amplitude errors, cavity/klystron failure, quadrupole misalignment errors, quadrupole gradient error, and beam-input mismatches.
\end{abstract}

\section{INTRODUCTION}

The current Los Alamos design for APT uses an integrated $\mathrm{NC} / \mathrm{SC}$ structure $[1,2]$. The $\mathrm{SC}$ structure starting at $217 \mathrm{MeV}$ accelerates the $100 \mathrm{~mA}$, cw proton beam to a nominal energy of $1.7 \mathrm{GeV}$. The beam is delivered onto a production target. through a high energy beam transport (HEBT) section [3].

One of the main advantages [1,4] of having a SC structure at high energies is that it allows a larger bore size reducing the risk of beam loss. In view of the importance of the beam-loss issue, we did study the effect of various types of error conditions and component failures on the beam. Of specific interest are the transverse beam-profile along the length of the linac and quality of the output beam which has direct impact on the transport of the beam through the HEBT.

The high-energy SC linac is comprised of two, medium and high $\beta$ sections with $\beta=0.64$ and 0.82 respectively. The bore radius in the sections are $6.5 \mathrm{~cm}$ and $8.0 \mathrm{~cm}$ respectively. Two design scenarios for the SC linac section are under consideration [1]. One uses SC quadrupole magnets in a FODO lattice; the other uses doublet room-temperature quadrupoles placed outside the cryostats. The results reported here correspond to the "singlet" SC design. Unless otherwise noted, simulations were done with a beam from the output of the NC structure at $217 \mathrm{MeV}$ which originated at the plasma surface of the ion source [5]. For the error studies, the beam was matched across the NC/SC transition by ramping the quadrupole-strengths down starting at 100 $\mathrm{MeV}$ without deliberate consideration of a currentindependent match described in Ref.1.

\section{MISMATCHES}

Setting errors for the quadrupole gradients, accelerating gradients, or cavity phases can produce beam mismatches. To study the effect of mismatch at the 217 $\mathrm{MeV}$ input point, we use an initial waterbag distribution (uniform filling of a 6-D phase-space ellipsoid) at 217 $\mathrm{MeV}$. The waterbag distribution has characteristics that are similar to those seen in real beams; the distribution in any 2-D projection is peaked in the center and falls off gradually at the edges. The mismatched input distributions are derived from the matched ellipses in all three phase-space planes; the ellipse parameters for the matched beam in each plane are scaled to adjust the beam size while leaving the rms-phase-space areas (emittances) constant. Equal mismatches were simultaneously applied in each plane, corresponding to $\mu=0.7$ and 1.3 , where $\mu$ is defined as the ratio of the initial $\mathrm{rms}$ beam size to the matched rms beam size.

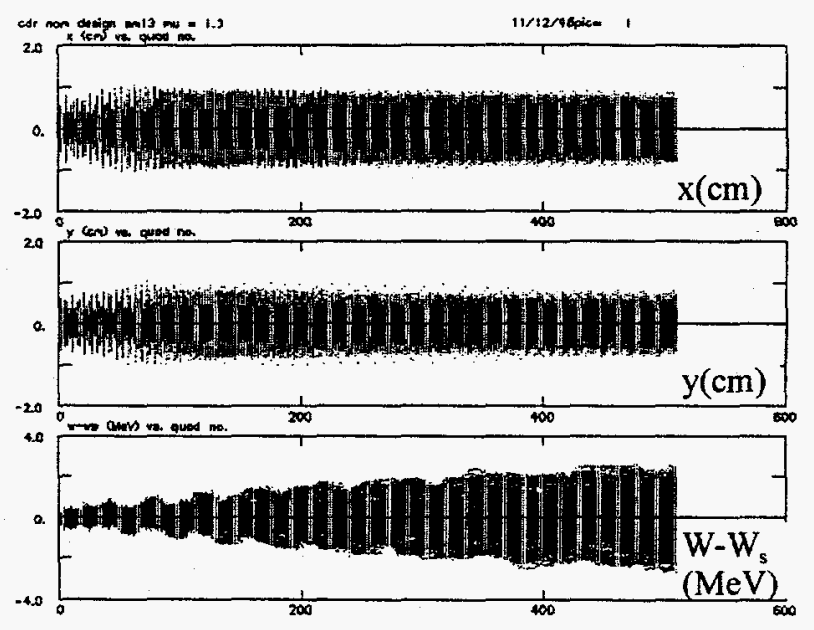

Figure 1. Transverse and longitudinal beam profiles vs. quadrupole number in the $\mathrm{SC}$ linac for a mismatched beam of $\mu=1.3$ at $217 \mathrm{MeV}$ with an initial waterbag distribution.

Figure 1 shows both the transverse and longitudinal profiles for $\mu=1.3$. The transverse beam size of the mismatched beam starts to grow immediately after the input, but after several periods settles down to a larger value. At the output end $(1.7 \mathrm{GeV})$ the $\mathrm{x}$ rms beam size grows to about $0.82 \mathrm{~cm}$ compared to about $0.46 \mathrm{~cm}$ for the matched case. The effect of mismatch in the longitudinal space is less pronounced. The output beam energy spread is only slightly larger when compared to the matched case.

The normal error-free output beam looks like a parabolic distribution in $\mathrm{x}$ or $\mathrm{y}$. The output distribution for a single point mismatch shows a shoulder on it that depends on the size of the mismatch. For this case, the extent of the distribution seems to be limited as demonstrated by adding the simulations of many 100,000 particle runs to reach several million total particles and as expected from the particle-core halo model [6]. The effect of mismatches for more than one point are still being studied.

\footnotetext{
* Work supported by the US Department of Energy
} 


\section{ERRORS}

Rf Covity Phase and Amplitude Errors

The rf Power system has feedback loops to maintain the phase and amplitude of the fields in the cavities. Because the klystron power is split to drive multiple cavities, the feedback loop adjusts the klystron phase and amplitude to control the average phase and amplitude error from the driven cavities. The result is that even though the average phase and amplitude of the cavities within an RF module can be controlled by the feedback loop to an average of $1.0^{\circ}$ in $\mathrm{rms}$ phase and $1 \%$ in $\mathrm{ms}$ amplitude, each individual cavity can have a larger variation. As a representative case, we have done simulations assuming $10^{\circ}$ and $10 \% \mathrm{rms}$ variations in each cavity with the average rms phase and amplitude for a klystron-driven set of cavities at $1.0^{\circ}$ and $1 \%$, respectively. The APT linac structures may experience about half of this level of RF amplitude and phase variations during normal operation.

We performed a set of 20 simulation runs, each with cavity phase and amplitude errors set randomly, assuming a uniform distribution within the above specified limits. The initial particle distribution was obtained from the preceding normal-conducting LE linac section. The results show that the output beam-energy centroid varies by about $\pm 4 \mathrm{MeV}$, which is acceptable for the HEBT. Transverse rms emittance varies by about $\pm 4 \%$. However, the longitudinal emittance growth, as expected, is higher than the error-free case by about $30 \%$.

\section{Quadrupole Magnet Misalignments}

Misalignments are simulated with a program, PARTREX that follows a central particle and a beam ellipse envelope through a sequence of elements using a sequence of matrix transformations in a linear-field approximation. Because the code transports beam ellipses rather than individual particles, it uses considerably less running time, enabling the user to make a statistical analysis of the beam behavior under random misalignments of the large number of quadrupoles in the linac. Each simulation corresponds to a complete set of random errors for each quadrupole in the linac.

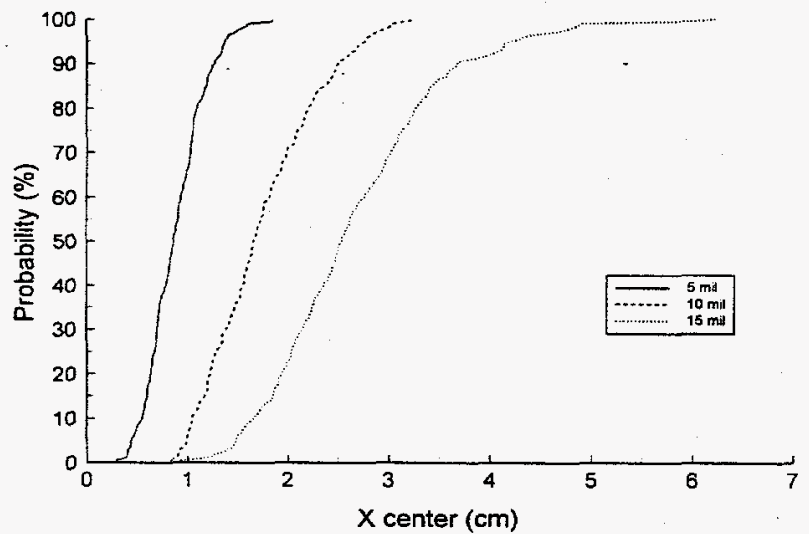

Figure 2. Beam centroid probability plots for $\mathrm{rms}$ quadrupole misalignment errors of 5,10 and $15 \mathrm{mil}$ (127, 254 , and $381 \mu \mathrm{m}$ ).
Two hundred simulations were made with random rms quadrupole alignment errors. Figure 2 shows the ordered-results for quadrupole misalignment errors of $0.127,0.254$ and $0.381 \mathrm{~mm}$. For a $0.254 \mathrm{~mm}$ rms random misalignment error, there is $95 \%$ probability that the beam-center shift will be less than $2.8 \mathrm{~cm}$. The corresponding numbers for $0.127-$ and $0.381-\mathrm{mm}$ misalignment errors are $1.4 \mathrm{~cm}$ and $4.2 \mathrm{~cm}$, respectively.

A $0.254-\mathrm{mm}$ rms uncertainty is a value that has been achieved for SC magnets in other accelerator facilities. A corresponding shift of $2.8 \mathrm{~cm}$ or larger with $5 \%$ probability suggests that provision should be made for steering in or after the linac. It should be noted, however, that the beam easily cleared the bore of the linac without steering for all the runs.

\section{Practical Set of Machine Errors}

We considered a set of realistic errors that include magnet misalignment in displacement, tilt, and roll, quadrupole gradient error, and RF phase and amplitude variations. The numbers used are uniform distribution of errors that have the following RMS widths:

- quadrupole magnet gradient error, $\pm 1 \%$

- quadrupole magnet tilt error, \pm 0.3 degree

- quadrupole magnet roll error, \pm 0.3 degree

- average RF phase error, \pm 1 degree

- average RF gradient error, $\pm 1 \%$

- local RF phase error, \pm 10 degree

- local RF gradient error, $\pm 10 \%$

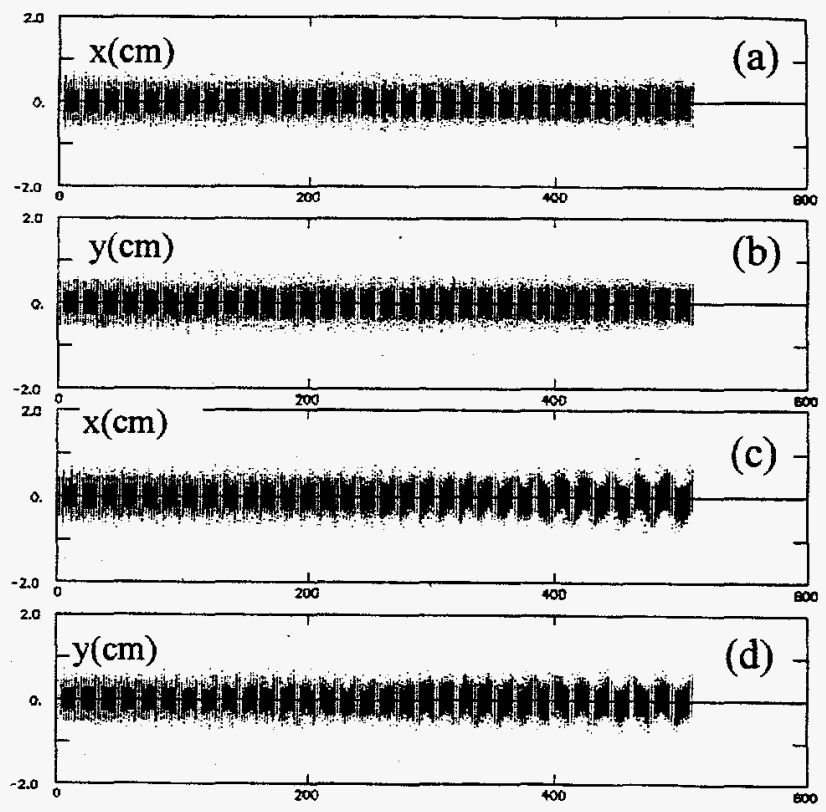

Figure 3. Transverse ( $x$ and $y$ ) vs. quadrupole number with (a-b) no errors and (c-d) realistic set of errors noted in the text.

Figures 3 (a) and (b) show the $x$ and $y$ profile plots of a normal beam. Corresponding profile plots with the above set of errors are shown in Figures 3 (c) and (d). A small centroid oscillation is observed while the longitudinal profile (not shown) does not show any significant effect. 


\section{FAULTS}

\section{RF Cavity/Klystron Failure}

If a single cavity or a klystron that powers several cavities fails, the beam-entry phase beyond the failure point will be shifted and, unless corrected, would generally result in poor acceleration efficiency and poor longitudinal focusing in the cavities downstream of the failure. Such an uncorrected situation could also lead to radial loss of beam. However, rephasing the linac beyond the point of failure is practicable because the cavities in the $\mathrm{SC}$ structure are electrically independent.

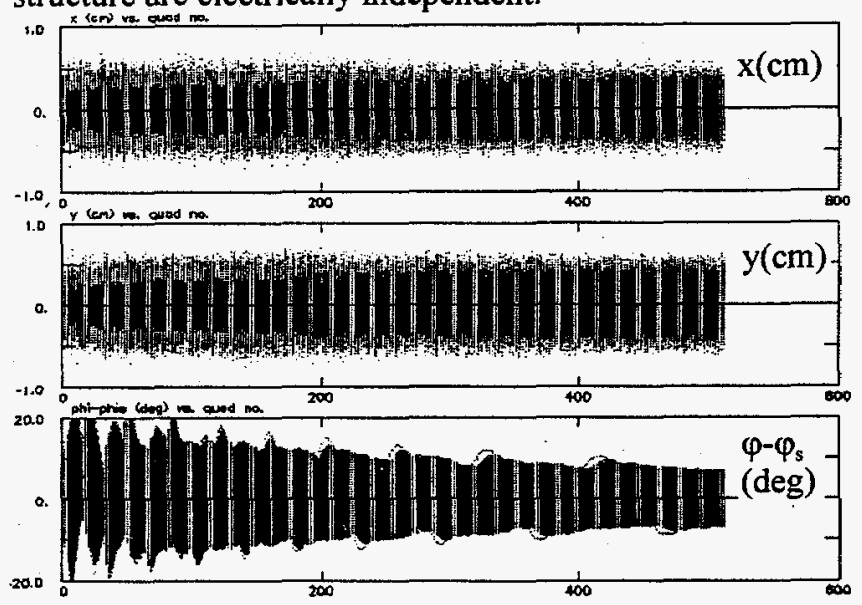

Figure 4. Beam profiles vs. Quadrupole number in the SC linac with the first klystron in the $\beta=0.64$ section turned off.

If the $r f$ amplitudes beyond the failure point are not reset, the output centroid energy will be reduced. As expected, the increase in longitudinal emittance is significantly larger than the corresponding increase of the transverse emittance. Also, a larger increase in longitudinal emittance is associated with cavity or klystron failures at the lower-energy end of the linac. This is due to the fact that in the lower-energy part of the SC linac, the longitudinal focusing loss as a result of a single cavity failure is more important, especially for compensation of the space-charge forces.

Figure 4 shows the beam profiles along the length of the SC linac when the first klystron in the $\beta=0.64$ section is turned off. There is a large increase in the longitudinal emittance. However, since the longitudinal emittance is of little concern to the HEBT/ Expander system, operation of the linac with a klystron off is not a problem in terms of beam at the target. Though not a major effect on the transverse emittances, this does, however, represent a mismatch with some attendant increase in the tails of the particle distribution.

\section{Quadrupole Magnet Failures}

Failure of one or more quadrupole magnets causes an immediate transverse mismatch resulting in subsequent emittance and halo growth. Since the focusing lattice is a FODO sequence, it is not surprising that a failure of an odd number of adjacent magnets causes a larger disruption. A failed pair of magnets early in the machine did not result in beam loss in the linac but did result in emittance growth of nearly a factor of 13. Readjusting the four quadrupoles downstream of the failed ones, a rematch can be achieved that reduces the emittance growth to near a factor of two. The output phase-space distributions with and without errors are shown in Figure 5. There are more particles on the edges of the distribution compared with the error-free case even with quadrupole readjustment.
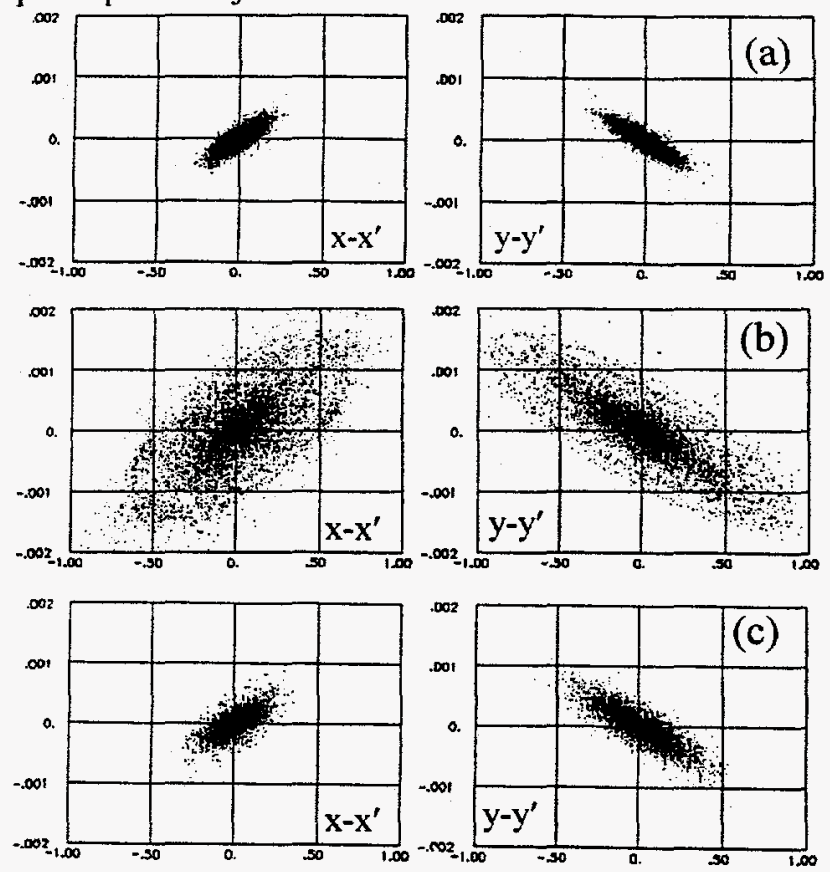

Figure 5. Output phase-space distributions with a pair of quadrupoles at the start of $\beta=0.64$ section turned off; (a) no failure, (b) with failure but no adjustment and (c) with downstream quadrupoles readjusted.

\section{CONCLUSION}

On the basis of the error studies completed, it can be concluded that none of the error conditions put the beamedge close to the aperture. Steering magnets in or after the linac should be installed for transporting the beam through the HEBT/Expander system. In case of quadrupole failure, retuning downstream of failed quadrupoles will be necessary for the beam to be transported by the HEBT/Expander system or the cryomodule can be replaced. HEBT can continue to operate with cavity/klystron failure.

\section{REFERENCES}

[1] S. Nath et al., "Beam Dynamics for the APT Integrated Linac," this conference.

[2] G. P. Lawrence and T. P. Wangler, "Integrated Normal-conducting/Super-conducting High power Proton Linac for APT," this conference.

[3] R. A. Shafer et al., "Overview of the APT HighEnergy Beam Transport and Beam Expanders," this conference.

[4] Los Alamos National Laboratory APT Conceptual Design Report, LA-UR-97-1329, April 1997.

[5] L. M. Young, "Simulations of the LEDA LEBT with $\mathrm{H}^{+}, \mathrm{H}_{2}^{+}$, and $\mathrm{e}^{-}$particles," this conference.

[6] T. P. Wangler et al., "New High Power Linacs and Beam Physics," this conference. 